\title{
Aplicación de biotecnologías para una mayor producción de terneros
}

\author{
Baruselli, P.S. ${ }^{\text {; }}$ Marques, M.O. ${ }^{2}$; Vieira, L.M. ${ }^{1}$; Konrad, J.L., ${ }^{3,4}$; Crudeli, G.A. ${ }^{3}$ \\ ${ }^{1}$ Dep. Reprod. Animal, Fac. Med. Vet. \& Zoot., Univ. San Pablo, Brasil; ${ }^{2}$ Geraembryo, Procópio, Brasil; \\ ${ }^{3}$ Cát. Teriogenología, Facultad. Cs. Veterinarias UNNE, Cabral 2139, Corrientes (3400), Argentina, \\ Tel/Fax 0379-4425753; ${ }^{4}$ CONICET, Argentina. Email: barusell@usp.br
}

\begin{abstract}
Resumen
Baruselli, P.S.; Marques, M.O.; Vieira, L.M.; Konrad, J.L.; Crudeli, G.A.: Aplicación de biotecnologías para una mayor producción de terneros. Rev. vet. 26: 2, 154-159, 2015. La incorporación de las técnicas de inseminación artificial y transferencia embrionaria (en ambos casos a tiempo fijo) y la aplicación de herramientas de sincronización y resincronización de celos, han demostrado ser capaces de aumentar la cantidad de terneros nacidos, incrementando por consiguiente la productividad y rentabilidad de las explotaciones ganaderas. Además, el empleo de semen y embriones de alta calidad involucra el avance genético de los rodeos. Se resalta que tales beneficios biotecnológicos requieren un adecuado control del rodeo, incluyendo la identificación de los vientres y la correcta ejecución de los procedimientos reproductivo, sanitario y nutricional.
\end{abstract}

Palabras clave: bovino, sincronización, inseminación, transferencia embrionaria.

\begin{abstract}
Baruselli, P.S.; Marques, M.O.; Vieira, L.M.; Konrad, J.L.; Crudeli, G.A.: Application of biotechnologies for the increment of calves' production. Rev. vet. 26: 2, 154-159, 2015. The incorporation of artificial insemination and embryonic transfer techniques (in both cases at fixed times) and the application of heat synchronization and re-synchronization tools have demonstrated to be useful methods to increase the number of calves born, consequently increasing the productivity and profitability of cattle exploitations. Furthermore, the use of semen and embryos involve the genetic improvement of the farms. Such biotechnical benefits require special features, with emphasis on the appropriate control of the rodeo that includes female identification and the correct execution of the reproductive, sanitarium and nutritional procedures.
\end{abstract}

Key words: cattle, synchronization, insemination, embryo transfer.

\section{Introducción}

En Brasil, a pesar de emplearse escasamente la inseminación artificial (IA), se verifica un considerable aumento en el porcentaje de hembras bovinas inseminadas, desde un 5\% en 2002 hasta aproximadamente un $10 \%$ en 2012. Con la intensificación del uso de IA, el país viene presentando un satisfactorio avance en el mejoramiento genético de los rodeos, por el incremento del número de terneros nacidos de toros genéticamente superiores. Asimismo se verifica que el aumento de la IA está asociado a un mayor empleo de los programas reproductivos que utilizan técnicas de sincronización para la inseminación artificial a tiempo fijo (IATF).

En el año 2013, alrededor de 8.750 .000 sincronizaciones para IATF fueron comercializadas en Brasil

Recibido: 3 agosto 2015 / Aceptado: 2 octubre 2015 (cifra estimada tomando en cuenta la venta de productos para sincronización). La Asociación Brasilera de Inseminación Artificial reportó la comercialización de 13.024.033 dosis de semen en el mismo año ${ }^{2}$. De esa manera, se calcula que el $67 \%$ de las IA realizadas en Brasil emplean técnicas de sincronización con IATF.

La IATF se basa en la utilización de fármacos comercialmente disponibles para sincronizar el ciclo estral y la ovulación de hembras bovinas, viabilizando la IA en momentos predeterminados, sin necesidad de la previa detección del celo. La técnica de IATF está bien establecida y los resultados son satisfactorios y previsibles. Esta biotecnología es capaz de sortear, con relativo éxito, los principales problemas que reducen la eficiencia reproductiva en hembras sometidas a programas de IA. Entre las dificultades, se pueden mencionar la escasez de mano de obra calificada en el campo, fallas en la detección del celo, elevado número de vacas 
en anestro posparto y vaquillas con atraso en la pubertad.

Luego de numerosos estudios para ajustar técnicamente los protocolos de IATF, surgió la necesidad de adecuarla a la rutina de manejo de los establecimientos ganaderos, especialmente a los objetivos específicos de los programas reproductivos de cada rodeo.

Dentro de las formas de intensificación del uso de la IATF, el establecimiento de programas de resincronización de las hembras no gestantes luego de la primera IATF merece una consideración especial. Este tipo de programa elimina la necesidad de observación del celo, reduce el número de toros necesarios para el repaso y principalmente, aumenta el número de productos nacidos de IA, que determinan un mayor aumento de productividad y mayor rentabilidad en la actividad.

En tanto, además del empleo de la IA para el mejoramiento genético, la utilización de la transferencia de embriones (TE), ha presentado un considerable aumento en Brasil, pasando de 200.000 embriones producidos en 2004 a 350.000 en $2011^{18}$. En la actualidad, la Sociedad Brasileira de Tecnología de Embriones estima que más de 400.000 embriones bovinos son producidos por año en ese país. La TE potencializa la ganancia genética por multiplicar tanto toros como hembras donantes genéticamente superiores. Los protocolos para TE a tiempo fijo (TETF) en receptoras de embriones, también fueron intensamente investigados y actualmente están siendo aplicados en numerosos establecimientos rurales.

Los requisitos necesarios para la utilización de TE en gran escala son semejantes a los requeridos para IA. Además de esto y debido a la dificultad en la criopreservación de embriones producidos in vitro $(90,7 \%$ del total de embriones producidos en Brasil), en la actualidad los programas de TE están limitados a la utilización de embriones no criopreservados.

En este sentido, varios grupos de investigación están trabajando para establecer un protocolo de criopreservación de embriones producidos in vitro e impulsar la utilización de la TE en gran escala. Adicionalmente, sumando las ventajas de ambas biotecnologías (IA y TE), las investigaciones han sido direccionadas para evaluar la eficiencia reproductiva y el incremento genético cuando las mismas son asociadas en establecimientos comerciales productores de carne.

El presente artículo tiene como objetivo evaluar el empleo de diferentes programas reproductivos que tratan de lograr la intensificación del empleo de la IATF y de la TETF, como así la asociación de esas biotecnologías en rodeos comerciales. Serán abordadas, principalmente, las técnicas que presentan impacto significativo en la eficiencia reproductiva de hembras de carne.

\section{Programas de resincronización en hembras de carne}

La resincronización es una tecnología que permite la realización de otra IATF en una hembra previamente inseminada. El objetivo de la técnica es aumentar el número de terneros nacidos de IA, eliminar la necesidad de determinar el estro, disminuir el número de toros para repaso, mejorar la eficiencia reproductiva y productiva de los rodeos y por último, aumentar el número de terneros genéticamente superiores.

Existen dos tipos de programas de resincronización, conforme al período en que el protocolo es iniciado; primero en el momento del diagnóstico de gestación, alrededor de 28 a 32 días después de la primera $\operatorname{IATF}^{3,9} \mathrm{y}$ segundo antes del diagnóstico de gestación, entre el día 19 y el día 23 después de la primera IATF $5,7,8,13,16,17$.

En Brasil, varios estudios fueron realizados para evaluar el inicio de la resincronización en hembras de carne 28 a 32 días posteriores a la primera IATF (A) en el día del diagnóstico de gestación y 22 días después de la primera IATF (B) antes del diagnóstico de gestación (Figura 1). La elección del momento para el inicio del protocolo de resincronización, dependerá del sistema de producción, del manejo de potreros y lotes en el establecimiento, de los costos de producción y de la mano de obra.

\section{Resincronización en el momento del diagnóstico de gestación}

La resincronización en el momento del diagnóstico de gestación proporciona mayor flexibilidad, pudiendo variar del día 28 a 32 después de la primera IATF, de acuerdo con la rutina y disponibilidad del veterinario
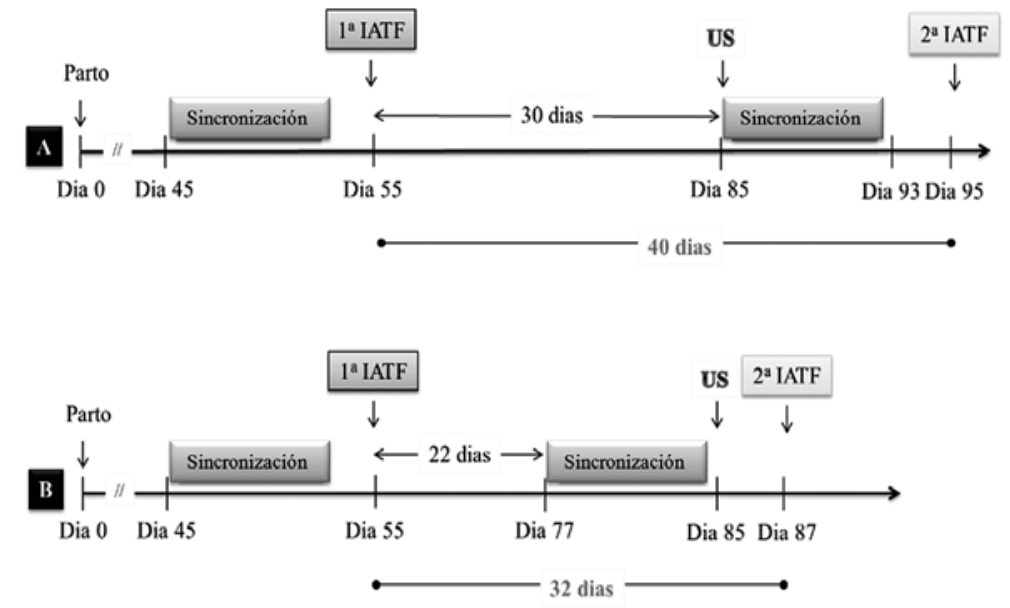

Figura 1. Diseño de los programas reproductivos utilizando la resincronización para IATF de hembras de carne. (A): inicio de la resincronización en el día del diagnóstico de gestación (28 a 32 días luego de la primera IATF), a los animales no gestantes. (B): inicio de la resincronización, en todos los animales sometidos a la primera IATF, 22 días después a primera IATF. Sincronización: protocolo de sincronización de la ovulación. IATF: inseminación artificial en tiempo fijo. US: diagnóstico de gestación por ultrasonografía. 


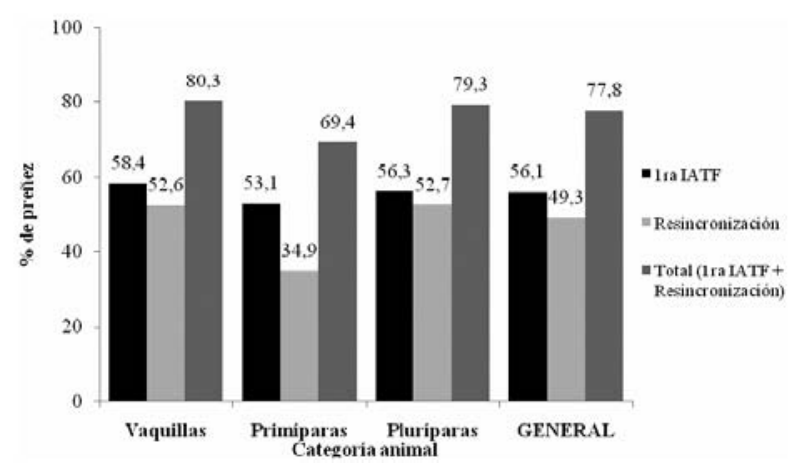

Figura 2. Porcentaje de preñez a la primer IATF y a la resincronización (segunda IATF) en vacas de carne (Bos indicus) de diferentes categorías. Para todas las categorías el intervalo entre IA fue de 40 días. Datos de 9.717 sincronizaciones para IATF durante la época de servicio de 2009/2010 y 2011/2012.

y de los empleados de la propiedad. Con esta metodología, el tratamiento de resincronización es realizado solamente en las hembras no gestantes (atrasando ocho días en relación a la opción B; Figura 1).

Frente a los beneficios adquiridos con el uso de la resincronización en el momento del diagnóstico de gestación, esta estrategia reproductiva viene siendo cada vez más empleada por los profesionales del sector. Datos con un significativo número de animales resincronizados $(\mathrm{n}=9.717)$ fueron analizados por un integrante de este grupo de trabajo, verificándose un $56,1 \%$ de preñez a la primer IATF y un $49,3 \%$ en la segunda IATF (resincronización 30 días después IATF previa), totalizando $77,8 \%$ de preñez con dos IATF realizadas con intervalo de 40 días (Figura 2).

Por lo tanto, los datos de campo indican que cuando la resincronización es utilizada de manera apropiada y en condiciones propicias (nutrición, sanidad y manejo adecuados) es posible obtener aproximadamente $75 \%$ de preñez en los primeros 40 días de servicio (incluyendo todas las categorías animales), manteniendo el intervalo entre partos de los animales gestantes, próximo a los 12 meses.

Después de los resultados positivos del empleo de la IATF asociada a la resincronización, se evaluó la eficacia de la introducción de la tercera IATF (segunda resincronización, (Figura 3) en los programas reproductivos, manteniendo 40 días de intervalo entre inseminaciones. Los resultados obtenidos son indicativos de que la asociación de tres IATF en vacas Nelore (intervalo entre IA de 40 días; resincronización en el momento del diagnóstico de gestación), puede proporcionar una elevada eficiencia reproductiva, obteniendo una preñez cercana al $90 \%$ con 80 días de estación de servicio (Figura 4) y con un intervalo entre partos próximo a los 12 meses. Se destacan los resultados positivos obtenidos con esta tecnología en vacas primíparas (Figura 5), categoría que frecuentemente presenta una baja eficiencia reproductiva.

\section{Resincronización sin previo diagnóstico de gestación}

La resincronización iniciada 22 días después a la IATF (sin diagnóstico previo de gestación, Figura 1B) proporciona anticipación de ocho días en la realización de la segunda IATF (comparada con la resincronización con inicio en el momento del diagnóstico de gestación, Figura 1A), aumentando las chances que la hembra pueda preñarse en los primeros 32 días de la estación de servicio. Esta tecnología puede ser de gran interés para establecimientos que venden los terneros al destete o que tengan una fecha fija para la comercialización de los animales productos de la resincronización. Por otro lado, el inicio del protocolo antes del diagnóstico de gestación (con 22 días) incluye a la sincronización del $100 \%$ de los animales (gestantes y no gestantes), previamente inseminados a tiempo fijo.

Los productos utilizados para la resincronización de las hembras son semejantes a los utilizados para la realización de la primera IATF. Entretanto, como los

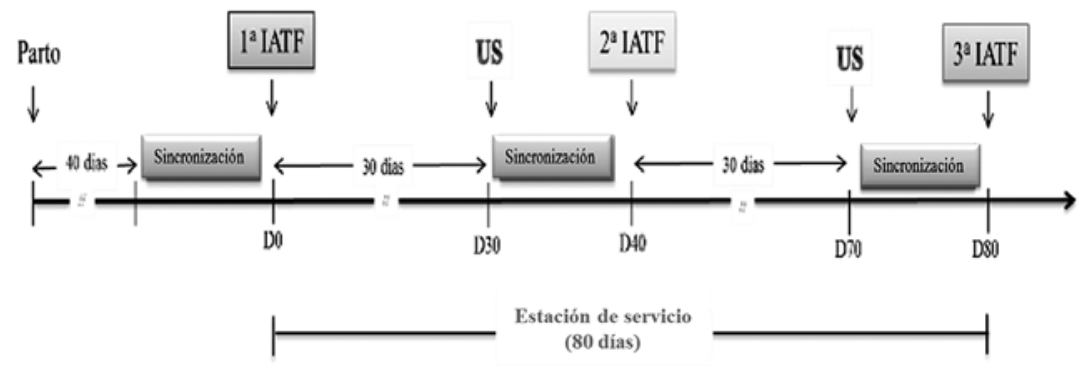

Figura 3. Diseño del manejo reproductivo con dos protocolos de resincroación (3 IATF con intervalo de 40 días entre inseminaciones) en 80 días stación de servicio (inicio de la resincronización en el momento del la ovulación; IATF: inseminación artificial en tiempo fijo; US: diagnóstico de gestación por ultrasonografía.

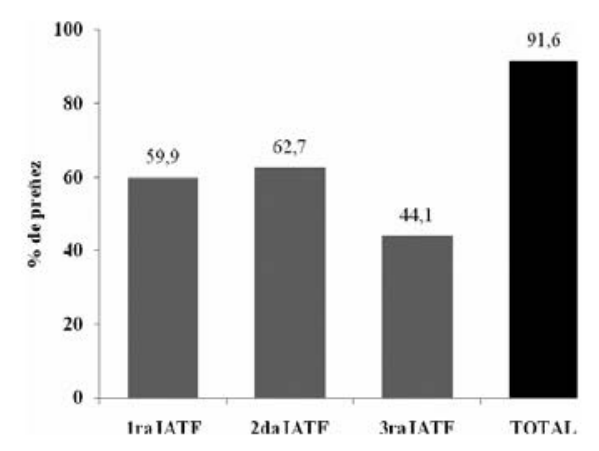

Figura 4. Porcentaje de preñez de vacas Nelore pluríparas $(n=1.365)$ sometidas a tres IATF (resincronización después del diagnóstico de gestación; 40 días de intervalo entre IATF), durante tres estaciones reproductivas, 2011/2014. 


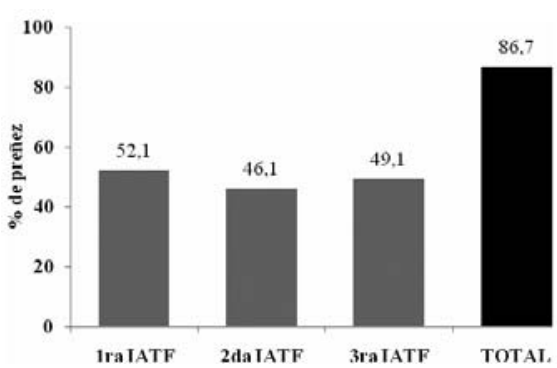

Figura 5. Porcentaje de preñez de vacas Nelore primíparas $(\mathrm{n}=678)$ sometidas a tres IATF (resincronización luego del diagnóstico de gestación; 40 días de intervalo entre IATF), durante a estación reproductiva 2013/2014.

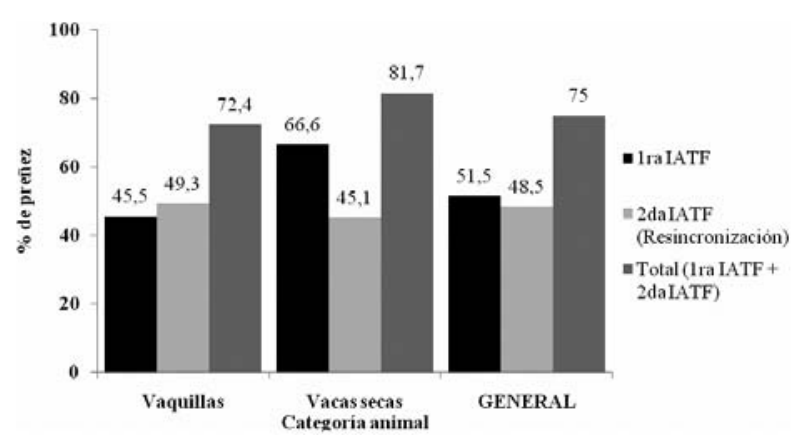

Figura 6. Tasa de preñez a la primera IATF y a la resincronización (segunda IATF) en vacas de carne (Bos indicus) de diferentes categorías (vaquillas y vacas secas). En las diferentes categorías el intervalo entre IA fue de 32 días. Datos de 540 sincronizaciones para IATF $^{16}$.

protocolos comúnmente utilizados en ganado de carne en América del Sur son en base a progesterona y estradiol, es importante resaltar que una de las funciones del estradiol es inducir la luteólisis ${ }^{1}$, pudiendo comprometer la gestación de los animales resincronizados. Debido a esto, se realizaron estudios para evaluar el porcentaje de preñez de hembras cebuínas resincronizadas con estradiol o GnRH en el día 22 post-IATF ${ }^{16}$.

En ese estudio, se observó un mayor índice de preñez en hembras (Bos indicus) resincronizadas con $1 \mathrm{mg}$ de benzoato estradiol $[49,2 \%(\mathrm{n}=140)]$, comparadas con las hembras resincronizadas con GnRH [37,2\% $(\mathrm{n}=137)]$, sin compromiso del porcentaje de preñez en referencia a la primera IATF [estradiol, $41,9 \%(\mathrm{n}=241)$ y GnRH, 41,5\% $(n=234)]$. También se verificó la pérdida gestacional al primer servicio (IATF) entre hembras sometidas a la resincronización con $1 \mathrm{mg}$ de benzoato estradiol en el inicio del protocolo de resincronización con intervalo de 32 días entre las dos inseminacio- nes $(4,1 \%, \mathrm{n}=195)$ o con monta natural $(2,0 \%, \mathrm{n}=198)$ para el repaso de las hembras no gestantes después de la primera IATF ${ }^{16}$.

De esta forma, el manejo de la resincronización con inicio en el momento del diagnóstico de gestación, así como la estrategia de resincronizar precozmente (antes del diagnóstico de gestación) viene siendo cada vez más empleado por los profesionales del sector. En un trabajo realizado con 540 hembras Nelore (387 vaquillas y 153 vacas secas) resincronizadas 22 días luego de la IATF (sin previo diagnóstico de gestación), se determinó un $51,5 \%$ de preñez a la primera IATF y un $48,5 \%$ de preñez a la resincronización ( $2^{\mathrm{a}}$ IATF), totalizando $75,0 \%$ de tasa de preñez con dos IATF realizadas con un intervalo de 32 días ${ }^{16}$ (Figura 6). Por lo tanto, los datos indican que cuando la resincronización precoz es utilizada en adecuadas condiciones, es posible obtener aproximadamente un $75 \%$ de tasa de preñez en apenas 32 días de estación de servicio (incluyendo todas las categorías animales), manteniendo el intervalo entre partos de los animales que se preñaron próximos a los 12 meses.

En otro trabajo se evaluó la utilización de tres IATF consecutivas con intervalo de 32 días entre inseminaciones (Figura 7) en vaquillas primíparas y pluríparas de la raza Nelore, con resincronización 22 días después a la IATF previa; antes del diagnóstico de gestación. En ese estudio, se registró un $83,1 \%$ de tasa de preñez en 64 días de estación de monta ${ }^{6}$ (Figura 8).

Con la implementación de programas reproductivos que utilizan la primera IATF precozmente (30 a 40 días pos-parto), seguida de dos resincronizaciones, se hace innecesaria la utilización de toros para repaso, ya que más del $80 \%$ de las hembras quedan gestantes por inseminación artificial, con una proyección de intervalo entre partos próxima a los 12 meses.

\section{Resincronización asociando IATF y TETF}

Como fue mencionado anteriormente, la TETF también puede ser una alternativa eficiente para la multiplicación de animales superiores, presentando índices

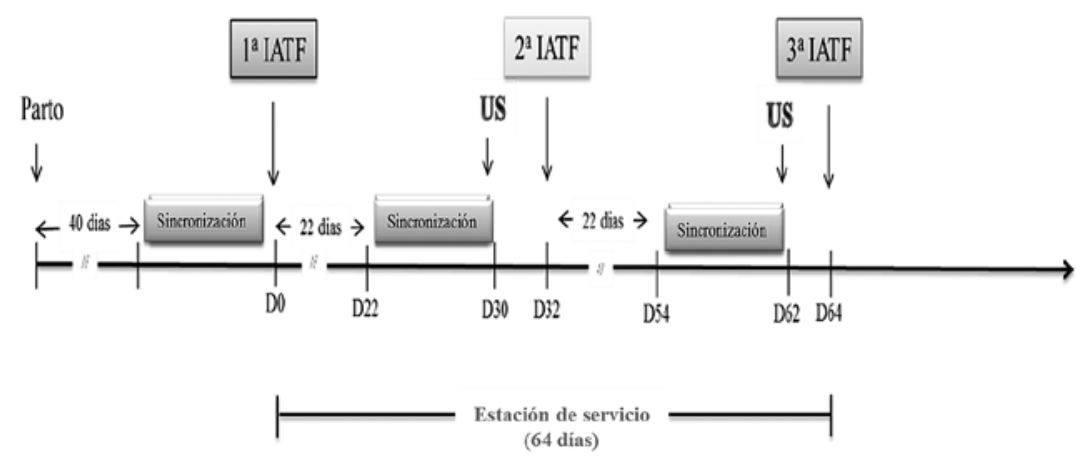

Figura 7. Diseño del manejo reproductivo con dos protocolos de resincronización (3 IATF con un intervalo de 32 días entre inseminaciones) en 64 días de estación de servicio (inicio de la resincronización antes del diagnóstico de gestación). Sincronización: protocolo de sincronización de la ovulación; IATF: inseminación artificial a tiempo fijo; US: diagnóstico de gestación por ultrasonografía. 
reproductivos que justifican su aplicación en rodeos de carne. De esta manera, frente a los resultados satisfactorios de los manejos de resincronización y el interés en incorporar las ventajas de la TETF, se realizaron estudios para evaluar el manejo de la resincronización asociando las biotecnologías de IATF y TETF.

En un trabajo se evaluó la eficiencia reproductiva de 634 vacas lactantes de la raza Nelore sometidas a uno de los siguientes cuatro manejos: dos IATF consecutivas (2IATF; $\mathrm{n}=160$ ), IATF seguida de TETF (IATF/TETF; $\mathrm{n}=160$ ), TETF seguida de IATF (TETF/IATF; $n=158$ ) y dos TETF consecutivas (2TETF; $n=156$ ). En todos los manejos, la resincronización fue realizada (con 40 días de intervalo entre inseminaciones) a los 30 y 23 días luego de la IATF y TETF, respectivamente. Los embriones fueron producidos in vitro y transferidos en fresco ${ }^{12}$ (Figura 9).

De esta forma y teniendo en cuenta el considerable aumento del mercado de producción in vitro de embriones bovinos, además de las estrategias de manejo, los investigadores también han desarrollado métodos para la selección de donantes con una muy alta capacidad de producción de embriones. Dentro de estos métodos, estudios recientes demuestran que la determinación de la concentración plasmática de la hormona anti-mulleriana (AMH) es un herramienta precisa y predictora de la cantidad de folículos antrales 4, 11, 14, 15 $\mathrm{y}$ consecuentemente, de la producción in vitro de donantes bovinas ${ }^{10}$. Por lo tanto, las concentraciones circulantes de AMH pueden ser utilizadas como un marcador para caracterizar donantes en cuanto a su eficiencia para la producción in vitro de embriones. El empleo de esa herramienta significaría un mayor número de embriones producidos por sesión de aspiración folicular y consecuentemente un mayor número de terneros producidos de toros y donantes genéticamente superiores.

Por fin, para que los beneficios de las biotecnologías sean viables, vale resaltar que es esencial una correcta determinación y ejecución de los procesos y procedimientos, incluyendo el adecuado sistema de control del rodeo, desde la identificación de los animales hasta el control preciso de los estados reproductivo, sanitario y nutricional.

\section{Colofón}

El empleo de las técnicas de IA y TE para multiplicar individuos superiores resulta en un significativo avance genético de los rodeos. En muchos estableci- mientos agropecuarios la aplicación de sendas técnicas aún presenta limitaciones, principalmente, debidas a las dificultades de ejecución del manejo y de la escasa disponibilidad de mano de obra calificada. El desarrollo y mejoramiento de las técnicas de sincronización para IATF y TETF, asociadas a los programas de resincronización, son herramientas aptas para alcanzar elevada eficiencia reproductiva de forma organizada, pre-determinada y con alta precisión. Por lo tanto, una vez establecido el objetivo de la actividad rural, la incorporación de los respectivos programas, aumenta la productividad y la rentabilidad del sistema de producción.

\section{REFERENCIAS}

1. Araujo RR, Ginther OJ, Ferreira JC, Palhão MM, Beg MA, Wiltbank MC. 2009. Role of follicular estradiol-17 
beta in timing of luteolysis in heifers. Biol Reprod 81: 426437.

2. Associação Brasileira de Inseminação Artificial (ASBIA). 2014. http://www.asbia. org.br/novo/informacoes/

3. Bartolome JA, Silvestre FT, Kamimura S, Arteche AC, Melendez P, Kelbert D, McHale J, Swift K, Archbald LF, Thatcher WW. 2005. Resynchronization of ovulation and timed insemination in lactating dairy cows I: use of the Ovsynch and Heatsynch protocols after non-pregnancy diagnosis by ultrasonography. Theriogenology 63 : 1617-1627.

4. Batista EO, Macedo GG, Sala RV, Ortolan MD, Sá Filho MF, Del Valle TA, Jesus EF, Lopes RN, Rennó FP, et al. 2014. Plasma antimullerian hormone as a predictor of ovarian antral follicular population in Bos indicus (Nelore) and Bos taurus (Holstein) heifers. Reprod Dom Anim 49: 448-452.

5. Chebel RC, Santos JE, Cerri RL, Galvão KN, Juchem SO, Thatcher WW. 2003. Effect of resynchronization with GnRH on day 21 after artificial insemination on pregnancy rate and pregnancy loss in lactating dairy cows. Theriogenology 60: 1389-1399.

6. Crepaldi GA, Freitas BG, Sá Filho MF, Guerreiro BM, et al. 2014. Reproductive efficiency of Nelore females submitted to three consecutive FTAI programs with 32 days of interval between inseminations. Anim Reprod 11: 355.

7. El-Zarkouny SZ, Stevenson JS. 2004. Resynchronizing estrus with progesterone or progesterone plus estrogen in cows of unknown pregnancy status. J Dairy Sci 87: 33063321.

8. Galvão KN, Sá Filho MF, Santos JE. 2007. Reducing the interval from pre-synchronization to initiation of timed artificial insemination improves fertility in dairy cows. $J$ Dairy Sci 90: 4212-4218.

9. Giordano JO, Wiltbank MC, Guenther JN, Pawlisch R, Bas S, Cunha AP, Fricke PM. 2012. Increased fertility in lactating dairy cows resynchronized with doubleOvsynch compared with Ovsynch initiated $32 \mathrm{~d}$ after timed artificial insemination. J Dairy Sci 95: 639-653.

10. Guerreiro BM, Batista EO, Sá Filho MF, Rodrigues CA, Castro A, Silveira CR, Bayeux BM, Dias EA, Monteiro FM, Accorsi M, Lopes RN, et al. 2014. Plasma antimullerian hormone: an endocrine marker for in vitro embryo production from Bos taurus and Bos indicus donors. Dom An Endocrin 49: 96-104.
11. Ireland JJ, Smith GW, Scheetz D, Jimenez-Krassel F, Folger JK, Ireland JL, Mossa F, Lonergan P, Evans AC. 2011. Does size matter in females? An overview of the impact of the high variation in the ovarian reserve on ovarian function and fertility, utility of anti-müllerian hormone as a diagnostic marker for fertility and causes of variation in the ovarian reserve in cattle. Reprod Fertil Dev 23: 1-14.

12. Martins CM, Reis PO, Vieira JH, Soares JG, Sá Filho MF et al. 2014. Effect of association of FTET and TAI in reproductive programs of Nelore females. Anim Reprod 11: 347.

13. Meyer JP, Radcliff RP, Rhoads ML, Bader JF, Murphy CN, Lucy MC. 2007. Timed artificial insemination of two consecutive services in dairy cows using prostaglandin F2alpha and gonadotropin-releasing hormone. J Dairy Sci 90: 691-698.

14. Monniaux D, Drouilhet L, Rico C, Estienne A, Jarrier P, Touzé JL, Sapa J, Phocas F, Dupont J, Dalbiès TR, Fabre S. 2012. Regulation of anti-müllerian hormone production in domestic animals. Reprod Fertil Dev 25: 1-16.

15. Rico C, Médigue C, Fabre S, Jarrier P, Bontoux M, Clément F, Monniaux D. 2011. Regulation of anti-müllerian hormone production in the cow: a multiscale study at endocrine, ovarian, follicular, and granulosa cell levels. Biol Reprod 84: 560-571.

16. Sá Filho MF, Girotto R, Santos FA, Sala RV, Barbuio JP et al. 2014. Resynchronization with unknown pregnancy status using progestin-based timed artificial insemination protocol in beef cattle. Theriogenology 81: 284-290.

17. Stevenson JS, Cartmill JA, Hensley BA, El-Zarkouny SZ. 2003. Conception rates of dairy cows following early not-pregnant diagnosis by ultrasonography and subsequent treatments with shortened Ovsynch protocol. Theriogenology 60: 475-483.

18. Viana JH. 2012. Levantamento estatístico da produção de embriões bovinos no Brasil em 2011: mudanças e tendências futuras.O embrião 6-10. 